\title{
Plastic behaviour and forming limit during biaxial compressions of magnesium alloy AZ31 at room temperature
}

\author{
I. Shimizu ${ }^{1, a}$ and N. Tada ${ }^{1}$ \\ ${ }^{1}$ Graduate School of Natural Science and Technology, Okayama University, 3-1-1 Tsushima-naka, \\ Kita-ku, Okayama 700-8530, Japan
}

\begin{abstract}
The plastic behaviour and compressive fracture of AZ31 magnesium alloy were examined at room temperature. The uniaxial compression tests and biaxial compression tests along linear and nonlinear strain paths were carried out until fracture using a unique biaxial compression testing machine developed by author. The results revealed the evolution of plastic anisotropy in biaxial compressions, that is the strain path dependency of work hardening. The adaptability of several forming limit criteria to the compressive fracture of AZ31 alloy was discussed. It was found that the uniform estimation of compressive forming limits by uniaxial and biaxial compressions was difficult by Freudenthal criterion and Oyane criterion. The result of Tresca energy criterion suggested that the criterion based on shear stress has possibility to predict the compressive forming limits at room temperature of AZ31 alloy.
\end{abstract}

\section{Introduction}

In recent years, magnesium alloys have been used in automobile, aerospace and computer industries, because of their lightweight, high specific strength, and good reusability. Most of magnesium alloys have hexagonal-close-packed structure; that causes unusual plastic behaviour due to deformation twinning. Therefore, energetic studies have been conducted on the characteristics of plastic deformation of magnesium alloys, e.g., influence of grain size on compressive behaviour [1], microstructure and texture evolutions during plane strain compression $[2,3]$ and hydrostatic extrusion [4], and so on. The deformation twinning also induces compressive fracture of magnesium alloys at room temperature. Barnett $[5,6]$ pointed out the strong influence of twins on the ductility of magnesium alloys by conventional tension and compression tests. The correlation of deformation twinning and room temperature formability of magnesium alloys has also been studied by AlSamman and Gottstein [7] under plane strain compression. However, the mechanical behaviour and formability in multi-axial compressive stress conditions have scarcely been investigated, nevertheless their importance for plastic working processes such as forging and extrusion.

The present study was undertaken to elucidate the biaxial compressive plastic behaviour and compressive forming limit of AZ31 magnesium alloy at room temperature using a unique biaxial

\footnotetext{
a e-mail : shimizu@mech.okayama-u.ac.jp
} 
compression testing machine developed by author [8]. The biaxial compression tests along linear and non-linear strain paths were conducted, thus the strain path dependencies of the mechanical properties and the forming limit were discussed. The investigation of compressive forming limit of magnesium alloys is still in the starting point, and the present study is probably the first one dealing with the influence of strain paths on the compressive forming limit.

\section{Experimental procedures}

The material employed in the present study is commercial quality AZ31 magnesium alloy $(\mathrm{Mg}-2.8$ wt $\% \mathrm{Al}-0.9 \mathrm{wt} \% \mathrm{Zn}$ ). The material was received as a plate of $15 \mathrm{~mm}$ thickness. The specimen was machined to cube shape with edge length of $7 \mathrm{~mm}$. The edges of the specimen were aligned along the thickness $(z)$, width $(y)$, and longitudinal $(x)$ directions of the plate. After surfaces of the specimen were mechanically polished to abrasive paper finish, the specimen was annealed at $523 \mathrm{~K}$ for $2 \mathrm{~h}$ in order to eliminate residual stresses. The resulting grains were rather equi-axed with the average grain size of about $180 \mu \mathrm{m}$.

The compression tests were performed using a unique biaxial compression testing machine. The mechanism for the biaxial compression is shown in Fig. 1. A rectangular block specimen is in the centre of the machine and contacting with four compression dies. Each die is attached to a linear slide unit, which enables the die movement in the direction parallel to the compressive axis. The compression in the horizontal direction is performed by dies 2 and 4, and dies 1 and 3 can move simultaneously in the horizontal direction. The compression in the vertical direction is performed by dies 1 and 3 in a similar way. Since the compressions in both directions can be independent each other, the biaxial compression with an arbitrary strain path is achieved. The detail of the testing machine is described elsewhere [8].

The basic mechanical properties and stress-strain relations of the AZ31 magnesium alloy were investigated by uniaxial compression tests in three perpendicular directions ( $x, y$, and $z$ directions). The biaxial compression tests were carried out along linear strain paths and along non-linear sequential plane strain paths without unloading. All tests were performed at room temperature until compressive fracture, with slow strain rate of $\sim 5 \times 10^{-4} \mathrm{~s}^{-1}$. A lubricant of boron nitride mixed with silicone grease was selected among several lubricants and was applied to the specimen/die interfaces to reduce friction. Figure 2 shows examples of fractured specimens after plane strain and equibiaxial compressions. The side faces of the specimen, namely free surfaces, were almost flat even after large compressive straining. This fact implies that the friction on specimen/die interfaces was sufficiently reduced by the lubricant.

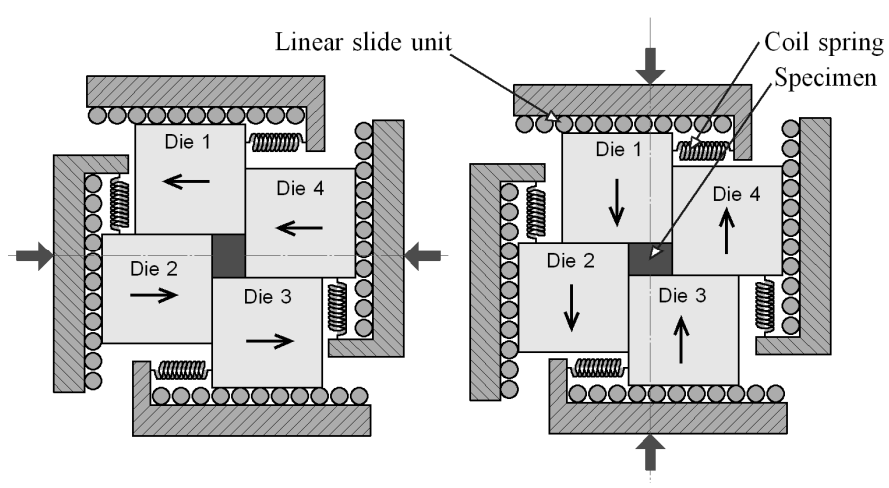

Fig. 1. Mechanism for biaxial compression.
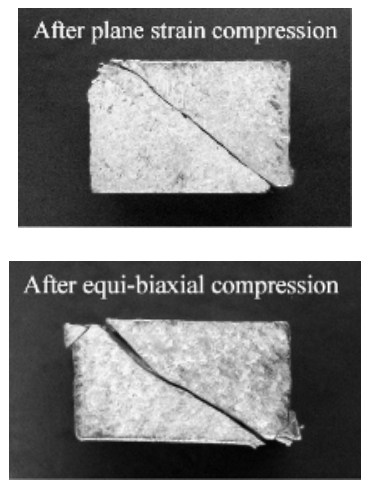

Fig. 2. Examples of fractured specimens after biaxial compressions. 


\section{Results and discussions}

\subsection{Compressive plastic behaviour}

True stress-strain curves obtained by uniaxial compression tests in $x, y$, and $z$ directions at room temperature are presented in Fig. 3. The good agreement of three curves implies that the initial condition of the material is almost isotropic. With increasing strain, the strain hardening rate becomes almost constant until the compressive strain $\varepsilon \approx-0.08$ and then it decreases again, like that of common metals. This variation of strain hardening rate is a feature of magnesium alloys and is caused by deformation twinning $[1,2]$, which is one of primary deformation mechanisms of AZ31 alloy at room temperature. However, the influences of the deformation twinning on the true stressstrain curves seem to be less than those presented by Barnett et al. [1], probably due to weak texture at the initial state. This initial isotropy of the material is useful for the investigation of strain path dependency.

The compressive plastic behaviour was investigated by biaxial compression tests along linear strain paths. The contours of equi-expenditure of plastic work introduced by Hill et al. [9] were evaluated by compressions along various linear strain paths to examine the change of yield surfaces and hardening behaviour with straining, as shown in Fig. 4. The equi-work contours were found to deviate from the isotropic von Mises yield curves with the progress of plastic deformation. This fact implies that the anisotropic hardening, namely plastic anisotropy, develops in the early stage of biaxial compressions. The variation of the plastic anisotropy is qualitatively agree with the texture changes shown by Yi et al. [10]; the texture evolves by tensile deformation within a small tensile strain of less than 0.1 . The texture change is caused mainly by the deformation twinning, which accompanies the orientation change in twined areas. In order to confirm the anisotropy evolution by biaxial compressions, the uniaxial compression tests were carried out for the pre-strained specimens by plane strain compressions in $x$ direction to the compressive strains $\varepsilon_{x 0}=-0.075$ and $\varepsilon_{x 0}=-0.15$. The obtained true stress-strain relations are shown in Fig. 5. When the compressive direction in the uniaxial compression was unchanged ( $x$ direction) with that in the preceding plane strain compression, the strain hardening rate continued to decrease. Contrary to that, increase in the strain hardening rate appeared when the compressive direction was changed to $y$ or $z$ direction. These remarkable differences in the stress-strain relations also prove the anisotropy evolution in the early stage of biaxial compressions.

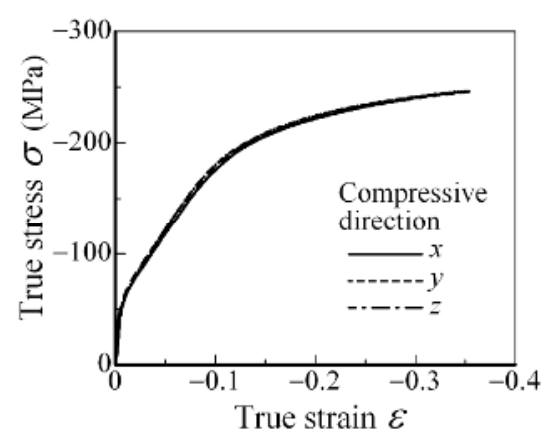

Fig. 3. True stress-strain curves of AZ31 specimens by uniaxial compression tests in $x$, $y$, and $z$ directions.

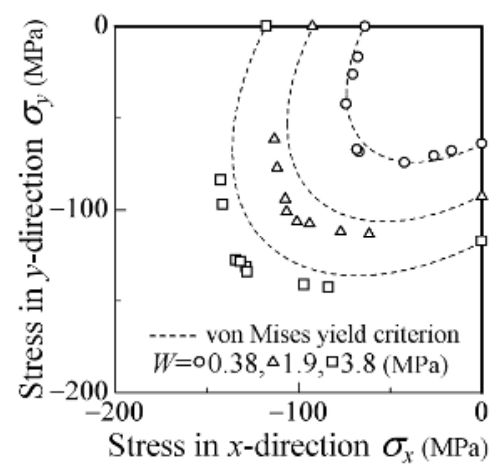

Fig. 4. Contour plots of equi-expenditure of plastic work $W$ obtained by biaxial compression tests along linear strain paths. 

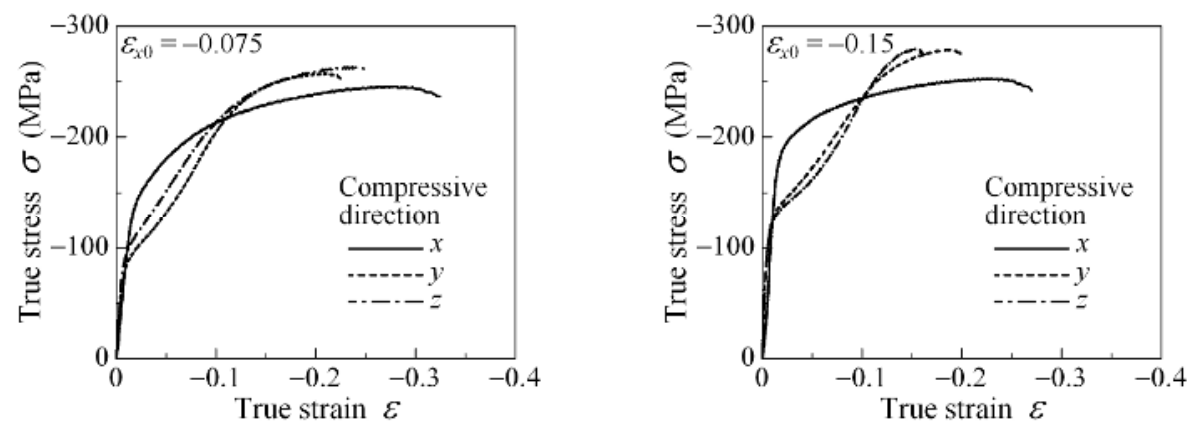

Fig. 5. True stress-strain curves by uniaxial compression tests of specimens pre-strained by plane strain compressions in $x$ direction to the compressive strain of $\varepsilon_{x 0}=-0.075$ and -0.15 .

\subsection{Compressive forming limit}

Figure 6 shows the nominal stress-strain curves by uniaxial compressions. The compressive fracture occurred when $d \sigma_{n}>0$ without any precursory phenomenon such as an area contraction, as seen also by the fractured shape shown in Fig. 2. From this fact, we can conclude that the compressive forming limit is almost coincident with the compressive fracture limit. At first, the compressive forming limits were plotted as major strain-minor strain relations and shown in Fig. 7. In this figure, the major strain is the maximum compressive strain, while the minor strain is the minimum compressive strain in compression-compression domain and the minimum tensile strain in compression-tension domain. The relatively good correlation in the results represents the clear strain path dependency of the compressive forming limit of AZ31 alloy.

The compressive forming limits at room temperature were investigated by means of typical forming limit criteria. Among several criteria, three of them, which were supposed to be applicable to the biaxial compressive conditions, were selected. Freudenthal [11] introduced a fracture criterion based on critical plastic work per unit volume,

$$
\int_{0}^{\bar{\varepsilon}_{f}} \bar{\sigma} d \bar{\varepsilon}=C_{1}
$$

where $\bar{\sigma}$ is the effective stress, $\bar{\varepsilon}_{f}$ is the effective strain at fracture and $C_{1}$ is constant. Oyane [12] suggested a criterion based on a void growth model,

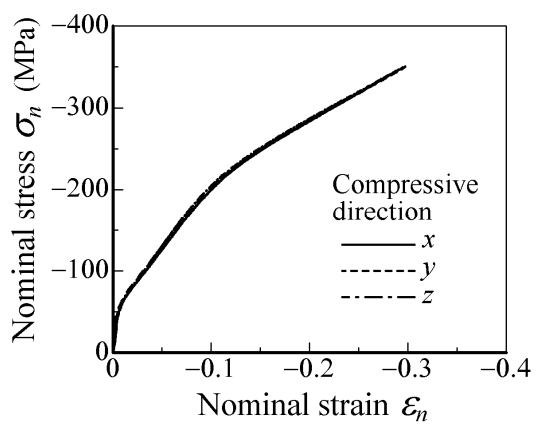

Fig. 6. Nominal stress-strain curves by uniaxial compressions.

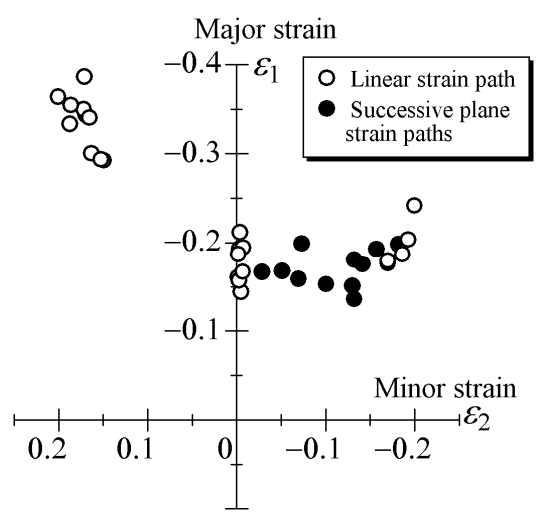

Fig. 7. Forming limits by uniaxial compressions and biaxial compressions along linear strain paths and along successive plane strain paths. 


$$
\int_{0}^{\bar{\varepsilon}_{f}}\left(1+\frac{\sigma_{m}}{A \bar{\sigma}}\right) d \bar{\varepsilon}=C_{2}
$$

where $A$ is a material constant and $\sigma_{m}$ is hydrostatic stress. The classical Tresca energy criterion was also selected,

$$
\int_{0}^{\bar{\varepsilon}_{f}} \frac{\left(\sigma_{\max }-\sigma_{\min }\right)}{2} d \bar{\varepsilon}=-\int_{0}^{\bar{\varepsilon}_{f}} \frac{\sigma_{\min }}{2} d \bar{\varepsilon}=C_{3}
$$

where $\sigma_{\max }$ is the maximum tensile stress (equals to zero in case of biaxial compressions) and $\sigma_{\min }$ is the maximum compressive stress.

Figure 8 shows Freudenthal criterion evaluated for the uniaxial and biaxial compression tests, plotted against the major strain $\varepsilon_{1}$. Large scattering of the Freudenthal criterion implies the difficulty to predict the compressive forming limit of AZ31 alloy based on the plastic work. The values of Oyane criterion are presented in Fig. 9. There is large discrepancy between the results by uniaxial and biaxial compressions. The selection of the material constant $A=3.0$, which was determined by two results of uniaxial and biaxial compression tests, is one important factor in Oyane criterion. In literatures, e.g. [13], $A=2 / 3$ was often used as the mean value between uniaxial and triaxial stressstates. However, the discrepancy was found to be larger with smaller value of $A$. This implies the difficulty to solve the discrepancy by the selection of the constant $A$, since the large value of $A$ weaken the effect of volumetric stress. In contrast, no discrepancy appeared between the results by uniaxial and biaxial compressions in Tresca energy criterion as shown in Fig. 10, though a certain degree of scattering remains in the results of biaxial compressions.

The fractured shapes of specimens shown in Fig. 2 denoted that the direction of crack was inclined at an angle of about $45 \mathrm{deg}$ to the major compression axis. A micrograph of fracture surface shown in Fig. 11 indicates that the morphology of the fractured surface has strong directionality due to the shear deformation. These two facts suggest that the shear stress is one major factor that initiates compressive fracture of AZ31 alloy. This is probably the reason why Tresca energy criterion yields better results than Freudenthal criterion and Oyane criterion. However, additional research is still necessary on the scattering of Tresca energy criterion in the biaxial compression region.

\section{Conclusions}

The elucidations of plastic behaviour and forming limit of material are indispensable for the success

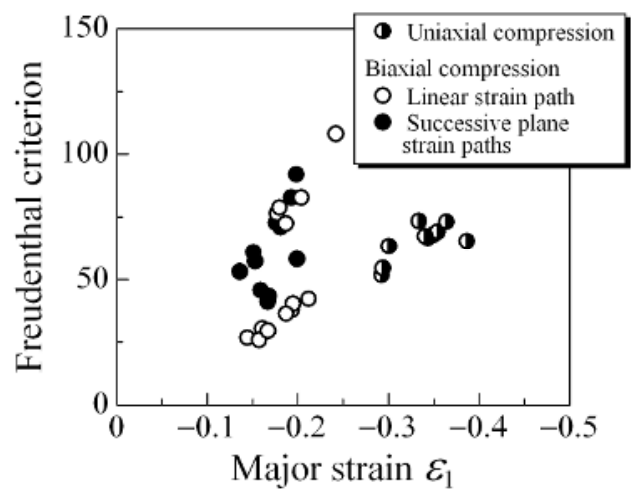

Fig. 8. Freudenthal criterion at compressive fracture of AZ31 alloy.

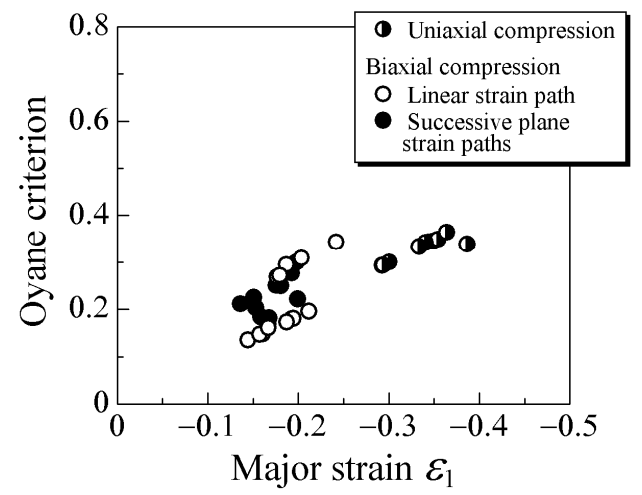

Fig. 9. Oyane criterion at compressive fracture of AZ31 alloy $(A=3.0)$. 


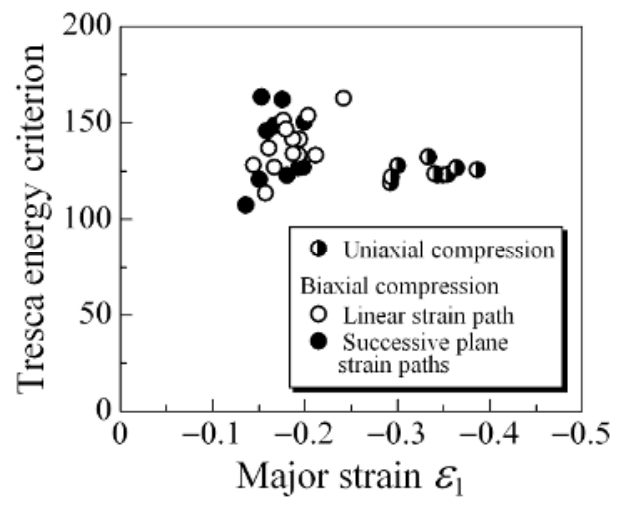

Fig. 10. Tresca energy criterion at compressive fracture of AZ31 alloy.



Fig. 11. Magnified fracture surface image after plane strain compression of AZ31 alloy.

of metal forming processes. In the present study, the uniaxial and biaxial compression tests of AZ31 magnesium alloy were carried out at room temperature, and the influences of strain paths on the plastic behaviour and compressive forming limit were discussed. The main conclusions are follows.

(1) The plastic anisotropy was found to develop rapidly in the early stage of biaxial compressions, due to limited number of slip systems and deformation twinning of AZ31 alloy.

(2) The compressive fracture of AZ31 alloy occurred without any precursory behaviour. The direction of the crack propagation was inclined at an angle of about $45 \mathrm{deg}$ to the major compression axis.

(3) Tresca energy criterion was found to estimate the forming limits of AZ31 alloy by uniaxial and biaxial compressions better than Freudenthal criterion and Oyane criterion.

\section{Acknowledgements}

The author thanks Mr. Kosuke Nakayama, Graduate student, Okayama University, for his assistance in the experiments. This work was supported by the Japan Ministry of Education, Science, Sports and Culture, Grant-in-Aid for Scientific Research (C), 21560094, 2009.

\section{References}

1. M.R. Barnett, Z. Keshavarz, A.G. Beer and D. Atwell, Acta Mater. 52, 5093 (2004)

2. R. Gehrmann, M.M. Frommert and G. Gottstein, Mater. Sci. Eng. A395, 338 (2005)

3. D.W. Brown, S.R. Agnew, M.A.M. Bourke, T.M. Holden, S.C. Vogel and C.N. Tomé, Mater. Sci. Eng. A399, 1 (2005)

4. J.Bohlem, S.B.Yi, J.Swiostek, D.Letzig, H.G.Brokmeier and K.U.Kainer, Scr. Mater. 53, 259 (2005)

5. M.R. Barnett, Mater. Sci. Eng. A464, 1 (2007)

6. M.R. Barnett, Mater. Sci. Eng. A464, 8 (2007)

7. T. Al-Samman and G. Gottstein, Mater. Sci. Eng. A488, 406 (2008)

8. I. Shimizu, Experimental Analysis of Nano and Engineering Materials and Structures 71 (2007)

9. R. Hill, S.S. Hecker and M.G. Stout, Int. J. Solids Struct. 31, 2999 (1994)

10. S.B. Yi, S. Zaefferer and H.G. Brokmeier, Mater. Sci. Eng. A424, 275 (2006)

11. A.M. Freudenthal, The inelastic behavior of solids (Wiley, New York, 1950)

12. M. Oyane, Bull. JSME 15, 1507 (1972)

13. A.V. Rao, N. Ramakrishnan and R.K. Kumar, J. Mater. Process. Technol. 142, 29 (2003) 